\title{
Gestionar la Autonomía Pedagógica: Un Factor de Calidad Reconocido
}

\section{Manage the Pedagogical Autonomy: A Recognized Quality Factor}

\author{
$\mathrm{M}^{\mathrm{a}}$ Antonia Casanova \\ Universidad Camilo José Cela, España
}

\section{DESCRIPTORES:}

Autonomía

Inclusión

Formación

Liderazgo

Evaluación

\section{RESUMEN:}

La sociedad actual se desenvuelve en contextos democráticos que se basan en la valoración y el respeto a la diferencia, como fuente de enriquecimiento mutuo y de progreso global. En consecuencia, se reconoce la diversidad como característica inherente a las personas, lo cual, trasladado a los centros docentes y a las aulas, exige un modelo de educación inclusiva, capaz de formar a las personas diferentes en unos mismos grupos, pero respondiendo a las singularidades de cada una de ellas. Es la fórmula válida para que se dé el mutuo conocimiento y desaparezcan los prejuicios derivados de la segregación durante las etapas de educación obligatoria. Para implementar las respuestas idóneas en cada centro docente, se requiere de autonomía pedagógica, organizativa y de gestión en las instituciones educativas, que deben disponer de la posibilidad de tomar decisiones propias para adecuar el sistema establecido a las realidades contextuales y a la población particular que deben atender. El ejercicio de una autonomía comprometida y responsable será la base de una calidad educativa pertinente con los requerimientos actuales de la sociedad. Para ello, la formación de directivos y docentes, el liderazgo inclusivo y la evaluación institucional interna, constituyen elementos básicos que permitirán alcanzar los objetivos previstos para el individuo y para el conjunto de la sociedad.

\section{KEYWORDS:}

Autonomy

Inclusion

Training

Leadership

Evaluation

\begin{abstract}
:
Today's society develops in democratic contexts based on valuation and respect for difference, as a source of mutual enrichment and global progress. As a result, diversity is recognized as an inherent characteristic of people, which, transferred to schools and classrooms, requires a model of inclusive education, capable of training different people in the same groups, but responding to the uniqueness of each of them. It is the valid formula for mutual knowledge to be given and prejudices arising from segregation during the stages of compulsory education to disappear. To implement the right answers in each school, pedagogical, organizational and management autonomy is required in educational institutions, which must have the possibility of making their own decisions to adapt the established system to the contextual realities and the particular population that they must attend to. The exercise of committed and responsible autonomy will be the basis of an educational quality relevant to the current requirements of society. To this end, the training of managers and teachers, inclusive leadership and internal institutional evaluation, are basic elements that will allow to achieve the objectives envisaged for the individual and for the whole of society.
\end{abstract} Eficacia y Cambio en Educación, 19(2), 9-22. https://doi.org/10.15366/reice2021.19.2.001 


\section{Introducción}

En los diferentes estudios de evaluación realizados a lo largo de las últimas décadas a nivel internacional (Davis y Thomas, 1999), la autonomía siempre aparece como factor de calidad para el funcionamiento y resultados de los Centros docentes. Parece lógico, considerando que, en cualquier empresa o institución, las decisiones más acertadas son las de las personas que dirigen su marcha, ya que conocen directamente las circunstancias que se atraviesan, el personal con que cuentan para su trabajo, los fines que pretenden a corto y largo plazo, las expectativas de la sociedad en relación con su quehacer, a la par que el contexto cercano y más o menos lejano en el que deben desenvolver su actuación en el momento presente y en el futuro.

Desde la distancia, la toma de decisiones es compleja, pues, por mucho que se pretenda entender una realidad, siempre será menos comprensible que cuando esta se vive en el día a día de la institución. Resulta obvio hasta hacer esta afirmación, aunque no lo parece tanto cuando muchos Centros educativos prefieren "obedecer" la normativa al pie de la letra y no asumir su responsabilidad ante los hechos que se producen y que deben resolverse con rigor, seriedad y profesionalidad. Tres características que se suponen al conjunto de docentes que componen el sector básico y principal de la comunidad escolar.

Sin embargo, el desempeño de esta autonomía exige, cómo no, la formación profesional docente adecuada, en este caso técnico-pedagógica, para aplicarla con la responsabilidad suficiente, de manera que surta los efectos positivos que se esperan de su ejercicio, por parte de la sociedad y de las Administraciones educativas. Dentro de esta formación -tanto inicial como permanente- es posible distinguir los aspectos que hacen alusión a los ámbitos puramente directivos (de gobierno, de gestión...) de la institución, como a los estrictamente didácticos, ya que la autonomía afecta al conjunto de la actuación institucional y no se deben separar ni aislar unos de otros. Ambos constituyen el quehacer global que lleva a cabo cada centro docente y que derivará en una óptima acción educativa o en unos resultados no deseados.

El liderazgo de la dirección (individual o colegiada), por ejemplo, supone una condición imprescindible para la puesta en práctica de cualquier modelo educativo acordado y para la toma de decisiones pertinentes en cada situación que así lo precise. La buena formación del profesorado en cada una de las áreas de trabajo que desarrolle, en otro sentido, resulta igualmente fundamental para conseguir las metas últimas y los objetivos cercanos del sistema, que llegará, o no, al alumnado en función de esa calidad del profesorado tan reclamada en los últimos tiempos.

Evidentemente, cada modelo de sociedad requiere de un modelo de educación que lo sustente, lo cual se comprueba claramente en la evolución de los diseños curriculares de las diferentes etapas históricas, también fácilmente constatable en los de las últimas décadas del pasado siglo y en los iniciales del actual. Los gobiernos democráticos exigen escuelas con currículos abiertos, flexibles, inclusivos, que hagan realidad las aspiraciones sociales del momento. Esta realidad que era muy distinta en épocas no tan pasadas, que aún perviven en determinadas escuelas que, a pesar de la normativa vigente, siguen aferrándose a prácticas obsoletas que debieran estar ampliamente superadas.

A lo largo de este artículo haremos referencia a los aspectos hasta aquí mencionados, intentando justificar la propuesta de dotar a los centros de una autonomía suficiente como para poder adaptar a sus contextos concretos las normas generales emitidas, que, por otra parte, deben garantizar y avalar la calidad educativa para toda la población nacional, al margen del territorio en que tenga lugar su escolarización.

\section{Un modelo educativo para la sociedad democrática}

Sociedad y educación constituyen dos caras de una misma moneda, complejas en su configuración, pero incuestionables ante cualquier enfoque o línea de razonamiento que se siga. La sociedad organiza un modelo de educación en función de los objetivos que quiere conseguir para sus ciudadanos, con el perfil de persona que considera adecuado para el momento en que se vive. La educación, por su parte, recoge el contexto en el que se desarrolla, pero aspira a más, a configurar una personalidad que mejore la realidad existente. Transmite valores del entorno y, a la vez, desea superarlos para alcanzar metas más equitativas para el conjunto de la población. 
Examinemos las características propias de una democracia, para concluir en el modelo más adecuado de sistema educativo, de manera que este ofrezca respuestas realmente válidas para vivir en nuestra sociedad actual.

\subsection{La sociedad democrática actual}

Cuando hablamos de sociedad democrática nos estamos refiriendo a un modelo de gobierno que respeta las diferencias, que las valora y las estimula, que busca y exige la participación ciudadana, que promueve la igualdad de oportunidades y la equidad social, que favorece la educación permanente... (Moya, 2015). Todo ello en aras del cumplimiento de sus principios básicos de configuración, en los que el compromiso de la población para la elección de sus gobiernos representativos es la base de su existencia. Una población responsable debe ser una población formada.

En consecuencia, con el paso de los años y de los avances ocurridos en todos los órdenes de la vida, se ha generado una realidad en la que podemos destacar las siguientes particularidades -ya comentadas, en parte, en una publicación anterior (Casanova, 2017)-que influirán en la decisión de uno u otro modelo educativo como el más apropiado para vivir y convivir en la diversidad, dentro de la sociedad democrática de nuestro tiempo:

a) Nos encontramos inmersos la sociedad de la información y del conocimiento, lo cual implica asimilar las informaciones recibidas, desagregarlas, compararlas, asumir las que resulten más coherentes, criticarlas, incorporarlas y transformarlas en conocimiento... La infoxicación es un peligro cierto para la ciudadanía

b) Los avances tecnológicos son permanentes y exigen, en muchos casos, estar al corriente de las numerosas novedades que se producen en cada momento, lo que requiere aprovechar los medios disponibles sin obsesionarse por poseer los últimos aparatos aparecidos en el mercado.

c) Las tecnologías de la información y la comunicación (TIC) han invadido nuestra privacidad, todos vivimos "en ellas", más o menos conscientemente. Están a nuestro lado y en todos los órdenes de la vida: personal, social, profesional. La información que se recibe a través de las mismas resulta inconmensurable. No hay, por lo tanto, falta de información, sino quizá exceso de datos, más o menos fiables. El dilema se plantea a la hora de valorarlos, por lo que los sistemas educativos, sobre todo en lo que se refiere a la selección de contenidos, debieran tomar nota para descargar los que no se consideren fundamentales.

d) Parece llegado el momento de desplazar el centro de interés desde el manejo de las Tecnologías de la Información y la Comunicación (TIC) al de las Tecnologías del Aprendizaje y del Conocimiento (TAC), que serán las que realmente hagan accesible la educación y la formación a la población a lo largo de la vida. No se debe conformar el sistema con la enseñanza del mero y superficial uso de instrumentos, sino que debe convertirlos en eje del progreso permanente de la persona.

e) La comunicación existente a nivel mundial deriva en una mayor movilidad individual y/o grupal, que resulta decisiva para la configuración, actual y futura, de la sociedad en numerosos países. Por razones políticas, bélicas, económicas, laborales, de estudios, de intereses personales..., la población migra y el entorno pasa a componerse de individuos pertenecientes a múltiples culturas e ideologías, que deben conocerse y convivir para salir adelante, juntos, en las mejores condiciones y oportunidades, ya que: "El pluralismo afirma y enriquece tanto a la sociedad como a sus integrantes, los ciudadanos" (Redondo, 2011). Esta realidad debe convertirse en un objetivo esencial de la sociedad democrática, si bien siempre respetando los principios éticos de conducta y la legalidad vigente de cada país. Según transcurren los años, cambian las circunstancias descritas. Debido a situaciones de violencia extrema en amplias regiones del mundo, son muchas las personas que emigran de sus países de origen para sobrevivir a las guerras y a las masacres, llegando con el estatus de refugiado a multitud de países. Se plantea, así, una nueva situación, desconocida por su magnitud (son millones las personas desplazadas), ante las sociedades democráticas, pues se deben ofrecer respuestas adecuadas para la totalidad de la población, tanto de origen como refugiada

f) La coexistencia multicultural caminando hacia la convivencia intercultural es otra realidad incuestionable, como consecuencia de las situaciones ya descritas y no solo en lo referente a las "culturas", aunque estas sean entendidas en sentido amplio, sino en todo lo relacionado con la convivencia entre las perso- 
nas diferentes (todas, al fin) en capacidades, talentos, intereses, motivaciones particulares, situaciones temporales específicas, etc. Esa es la sociedad democrática a la que se aspira y que exige seguir en ruta para lograrla.

g) El cambio acelerado de situaciones sociales y vitales es permanente. Es, al fin, lo único cierto de la situación: que se modifica día a día en múltiples aspectos debido, en gran medida, a lo apuntado en los apartados anteriores. De un día para otro se transforman numerosas opciones laborales concretas, cambian sistemas educativos que intentan mejorar sus resultados, medios técnicos hasta ayer válidos quedan desfasados, entornos personales que en pocos años han variado y ni se parecen a los que conocimos en nuestra infancia o juventud, nuevas posibilidades personales se abren ante nuestro horizonte, desaparecen otras que parecían ciertas y seguras... Esta es la tónica de la existencia actual y su exigencia educativa: el seguir aprendiendo a lo largo de la vida. Una vida de incertidumbre, frente a la seguridad, la certeza y el dogma que imperaba no hace tanto en el pasado siglo. En pocos años ha desaparecido la tierra que pisábamos y nos hemos quedado navegando "en un océano de incertidumbres a través de archipiélagos de certeza" (Morin, 2001, p. 104). Esta afirmación debería tener, también, repercusiones inmediatas en nuestros sistemas educativos, enseñando "principios de estrategia que permitan hacer frente a los riesgos, lo inesperado y lo incierto, y modificar su evolución en virtud de la información adquirida en el camino" (Morin, 2001, pp. 20-21), especialmente ahora, que todos buscamos una calidad que, inexplicablemente, nunca llega (Casanova, 2017, pp. 31-34).

h) Una sociedad mundial en situación de pandemia, confinada por la COVID-19. Esta es la última caracterización que puede citarse en el momento en que se escribe este texto y que, quizá, continúe cuando se publique. La presencia del coronavirus en nuestro entorno habitual ha trastocado toda la forma de relación social, educativa, laboral..., existente hasta el momento. Era inimaginable que pudiera producirse una circunstancia tal que, en la práctica totalidad de los países, y refiriéndonos específicamente a la educación, se cerraran las escuelas de un día para otro y fuera necesario modificar los planes de trabajo de docentes y escolares en todos los niveles, desde la Educación Infantil hasta la Universidad, pasando por las diversas modalidades autorizadas en el sistema educativo. Cierre del sistema que influyó decisivamente en la vida familiar, pues se trasladó, en buena parte, la acción docente del profesor a los padres y madres. En la mayoría de los casos no afectó al campo laboral, porque también cerraron muchas empresas de todo tipo o se pudo continuar mediante el teletrabajo, pero sí lo hizo cuando los padres o tutores de los niños tuvieron que salir de casa en horarios habituales. Una situación compleja que, efectivamente, cambió de modo radical el usual modelo educativo, rompiendo cualquier molde establecido hasta el momento.

\subsection{Consecuencias educativas de los planteamientos democráticos}

Fijándonos en cada uno de los puntos desarrollados en el apartado anterior, podemos concluir que son dos las exigencias globales para los sistemas educativos, si quieren ofrecer respuestas válidas para superar los retos que se plantean a la sociedad y a cada persona en particular: la adopción de un modelo de educación inclusiva y la necesidad inexcusable de dotar de autonomía suficiente a los centros docentes, para que puedan flexibilizar las normas generales emitidas por las Administraciones y convertir en accesible el diseño curricular para toda la población escolarizada.

\subsubsection{Educación inclusiva}

Sin extendernos demasiado en la primera propuesta realizada, es decir, en el modelo de educación inclusiva como el idóneo para conseguir una democracia plena -dado que este artículo hace alusión directa a la segunda: la autonomía-, sí parece oportuno destacar algunas de las razones evidentes que aconsejan esta opción como la más apropiada para nuestro mundo y el que parece que se avecina (Ainscow, 2014).

Antes de enumerar estas razones, digamos que se entiende por educación inclusiva la que apuesta por una escuela en la que se atiende a todo tipo de alumnado, sean cuales fueren sus características, ofreciendo respuestas diversificadas en función de la singularidad de cada uno de sus alumnos. Si todos los alumnos y alumnas son diferentes, no será razonable intentar que sean ellos los que se adapten al sistema, sino que, por el contrario, sea el sistema el que se flexibilice para atender a sus diferencias. Tampoco resulta funcional ni eficaz realizar las consabidas "adaptaciones curriculares", pues, si partimos del concepto de inclusión expuesto, sería preciso elaborar una adaptación para cada estudiante, dado que todos son 
distintos y presentan diversas peculiaridades. Lo razonable, por tanto, es adecuar el sistema en las aulas, para favorecer el aprendizaje de todos.

En síntesis, es posible concretar las ventajas de la inclusión en las siguientes:

- La sociedad no admite a quien no conoce. Si las personas diferentes deben convivir juntas, la mejor opción es que se eduquen juntas.

- La ética exige que el derecho a la educación en igualdad de oportunidades sea disfrutado por toda la población y es sabido que escuelas diferentes derivan en oportunidades diferentes. Esta garantía solo está avalada por la educación inclusiva.

- La accesibilidad curricular debe partir de la implementación del Diseño Universal para el Aprendizaje, con sus tres principios aplicables en cualquier nivel educativo: proporcionar múltiples medios de representación, proporcionar múltiples medios de acción y de expresión y proporcionar múltiples medios de motivación y compromiso.

- Las escuelas de carácter diferenciado no favorecen la posterior incorporación a la sociedad, en la que toda la población deberá trabajar cooperativamente para alcanzar las metas comunes.

- Los agrupamientos homogéneos no facilitan el aprendizaje de personas diferentes. La riqueza de la heterogeneidad es un factor de calidad reconocido en los resultados de estudios, investigaciones o trabajos de todo tipo (VV.AA., 2011).

- Los recursos educativos se han ampliado y han evolucionado de forma espectacular, lo que favorece su utilización para lograr una mejor adecuación educativa a cada escolar.

- En definitiva: la separación nunca favorece la inclusión, ni en la escuela ni en la sociedad.

\subsubsection{Autonomía institucional}

Aunque el texto que sigue abordará este epígrafe con detalle, pues es el objeto del monográfico en el que se publica, cabe anticipar unos comentarios como conclusiones previas a la exposición realizada hasta ahora.

Casi resulta obvio resaltar la necesidad de que los centros docentes posean capacidad de decisión, es decir, autonomía, para poder implementar el modelo educativo exigido en democracia (VV.AA., 2009). Hablar de calidad educativa y no delegar las decisiones pedagógicas en las instituciones que deben conseguirla resulta incoherente y obsoleto, pues pensar que todo se resuelve mejor desde un poder centralizado supone no entender la complejidad de la vida y de la educación, ambas contextualizadas en muy distintos entornos y vivencias. El conocimiento cercano, el dominio de esa complejidad solo se da en la propia institución en la que se producen los hechos educativos.

Todas las premisas expuestas hasta ahora exigen autonomía de gestión en los centros docentes. Si es imprescindible la convivencia en la diversidad y se admite que cada persona, cada alumno, es diferente al otro, se está reconociendo la necesidad de tomar medidas mediatas e inmediatas para ofrecer respuestas válidas en las circunstancias variadas que se presentan cada día en los centros y en las aulas. Parece un argumento suficiente para dotar de autonomía a las instituciones, de modo que puedan y sean capaces de ajustar lo establecido en las regulaciones de los sistemas a las peculiaridades que se les plantean en su quehacer habitual.

Un modelo inclusivo de educación, como el establecido y aceptado -al menos, teóricamente- por las normas vigentes en estos momentos, exige atender a la diversidad, lo cual alude directamente al ajuste organizativo, metodológico y evaluador en cada centro. Resultan factores de calidad relevantes para que los resultados obtenidos globalmente y también en particular por cada estudiante sean los esperados. La rigidez en la aplicación sistémica no ayuda a la mejora de los aprendizajes ni a la adquisición de competencias. Solo la adecuación diaria de la enseñanza a los muy distintos modos de aprender del alumnado 
dará la posibilidad de avanzar en el camino de la formación, lo cual implica la toma de decisiones autónomas en cada situación que lo precise. En primer lugar, conformando un proyecto educativo y curricular propio y, en segundo, optando en su aplicación por la oferta flexible en cada grupo, en función de los rasgos que presente.

\section{3. ¿De qué hablamos cuando nos referimos a la autonomía de los centros?}

De acuerdo con el Diccionario panhispánico del español jurídico (dpej.rae.es), se entiende por autonomía pedagógica de los centros: "Capacidad de los centros en la enseñanza no universitaria para adoptar experimentaciones, planes de trabajo, formas de organización, normas de convivencia y ampliación del horario escolar o del horario lectivo de áreas o materias, adopción de los libros de texto y demás materiales que hayan de utilizarse en el desarrollo de las diversas enseñanzas; todo ello en los términos que establezcan las administraciones educativas y sin que en ningún caso se impongan aportaciones económicas a las familias ni exigencias para las administraciones educativas".

Como es obvio, esta definición está contextualizada en lo regulado actualmente en la legislación educativa española, ya que la autonomía podría referirse a toda la actuación de los centros docentes, sin restricciones e, incluso en el caso que nos ocupa, también resulta más amplia cuando se hace alusión a los centros concertados y privados, que disponen de mayores atribuciones para organizar su funcionamiento, si bien siempre respetando los elementos básicos establecidos para el conjunto del Estado (titulaciones del profesorado, currículum básico, equipamientos e instalaciones, ratios, etc.), como garantía de calidad para toda la población y como base para la homologación de los títulos obtenidos en cualquier territorio del mismo.

Saliendo del estricto ámbito legislativo e incluso al margen de su regulación, se entiende la autonomía como la capacidad de decidir de la comunidad educativa de un centro docente en relación con las actuaciones que se consideran adecuadas para el buen funcionamiento de la institución, facilitando así la adaptación de los agrupamientos de alumnos, de la utilización de recursos disponibles, de la adaptación de los elementos curriculares, del uso de las instalaciones propias y del entorno... Es decir, la facultad que debe poseer toda organización para lograr los objetivos que se propone. Si no dispone de esta autonomía, no será responsable de los resultados obtenidos, lo cual exime de compromiso a sus protagonistas; en definitiva, cuando se toman decisiones autónomas, se requiere de su evaluación y de la rendición de cuentas ante la sociedad. En caso contrario, ¿quién se hará responsable del éxito o fracaso de un sistema educativo? ¿La Administración central? ¿La autonómica? Difícil, pues, como antes se comentó, la elección de opciones desde la distancia resulta altamente arriesgada para que sea atinada. La cercanía favorece el acierto, sin duda alguna.

\subsection{Lo que dice la legislación española}

Formalmente, se habla de autonomía institucional para su ejercicio desde los centros educativos a partir de la publicación de la Ley Orgánica 8/1985, de 3 de julio, reguladora del Derecho a la Educación (BOE del 4) (a partir de ahora, LODE), si bien este apunte inicial se ha completado posteriormente, concretando y ampliando los términos en que aquí aparecen.

En su artículo 15, la LODE determina: "En la medida en que no constituya discriminación para ningún miembro de la comunidad educativa y dentro de los límites fijados por las leyes, los centros tendrán autonomía para establecer materias optativas, adaptar los programas a las características del medio en que estén insertos, adoptar métodos de enseñanza y organizar actividades culturales escolares y extraescolares”.

La descripción de las facultades de autonomía que se otorgan a los centros, en este caso, resulta bastante genérica y, por otro lado, cita algunos ámbitos en los que desde siempre las instituciones educativas han gozado de la misma, como son la adopción de métodos y la organización de actividades escolares y extraescolares. Sí supone un avance la posibilidad de establecer materias optativas (hasta ese momento inexistentes) y la adaptación de los programas al contexto en el que realicen su trabajo. No fija los límites 
de esta adaptación, si bien esta opción está limitada por el establecimiento de unas enseñanzas básicas o mínimas desde la Administración (central o autonómica) que no pueden eludirse, por lo cual hay que suponer que se pueden ampliar o matizar en su práctica en las aulas, pero nunca suprimir los objetivos o contenidos entendidos como imprescindibles para que toda la ciudadanía cuente con igualdad de oportunidades al finalizar su educación obligatoria.

El diseño de la autonomía curricular, como se comprueba, requiere de una regulación legal que no abarque todo el currículum que deben implementar los centros, sino solamente una parte, fundamental, dejando margen de ampliación o adecuación para los distintos territorios que componen en Estado y para cada centro docente en particular. Esta situación no se produce en España hasta la publicación del Decreto 69/1981, de 9 de enero (BOE del 17), de ordenación de la Educación General Básica y fijación de las enseñanzas mínimas para el Ciclo Inicial, exigida por la transferencia de las competencias educativas al País Vasco y a Cataluña.

Tras esta normativa, la Ley Orgánica 2/2006, de 3 de mayo, de Educación (BOE del 4) (a partir de ahora, LOE), retoma la importancia de la autonomía dedicando su Título $\mathrm{V}$ a la participación, autonomía y gobierno de los centros docentes. Dentro de este Título, su Capítulo II aborda la autonomía de los centros. Así, en su artículo 120, de disposiciones generales, ordena:

- Los centros dispondrán de autonomía pedagógica, de organización y de gestión en el marco de la legislación vigente y en los términos recogidos en la presente Ley y en las normas que la desarrollen.

- Los centros docentes dispondrán de autonomía para elaborar, aprobar y ejecutar un proyecto educativo y un proyecto de gestión, así como las normas de organización y funcionamiento del centro.

- Las Administraciones educativas favorecerán la autonomía de los centros de forma que sus recursos económicos, materiales y humanos puedan adecuarse a los planes de trabajo y organización que elaboren, una vez que sean convenientemente evaluados y valorados.

- Los centros, en el ejercicio de su autonomía, pueden adoptar experimentaciones, planes de trabajo, formas de organización o ampliación del horario escolar en los términos que establezcan las Administraciones educativas, sin que, en ningún caso, se impongan aportaciones a las familias ni exigencias para las Administraciones educativas.

- Cuando estas experimentaciones, planes de trabajo o formas de organización puedan afectar a la obtención de títulos académicos o profesionales, deberán ser autorizados expresamente por el Gobierno".

Se completa el contenido de este artículo con el del 124, dedicado a normas de organización y funcionamiento: "1. Los centros docentes elaborarán sus normas de organización y funcionamiento, que deberán incluir las que garanticen el cumplimiento del plan de convivencia. 2. Las Administraciones educativas facilitarán que los centros, en el marco de su autonomía, puedan elaborar sus propias normas de organización y funcionamiento".

Además, en la disposición adicional cuarta, sobre libros de texto y demás materiales curriculares, se explicita: "1. En el ejercicio de la autonomía pedagógica, corresponde a los órganos de coordinación didáctica de los centros públicos adoptar los libros de texto y demás materiales que hayan de utilizarse en el desarrollo de las diversas enseñanzas”.

Evidentemente, entre la LODE y la LOE aparece un importante avance en la concreción de las posibilidades de dirección autónoma en los centros, si bien siempre con las limitaciones de las instituciones de carácter público, entre ellas una decisiva para la experimentación de determinados proyectos, como es la de elección de personal docente con la formación adecuada para llevarla a cabo. Es una realidad con la que hay que contar y que, por tanto, cualquier iniciativa innovadora que se ponga en marcha, requerirá de un buen liderazgo del equipo directivo para involucrar en la misma a la mayoría del profesorado que deberá participar en ella. 
Proyecto educativo, proyecto de gestión (económica), organización, funcionamiento, experimentación de programas, ampliación de horario..., ofrecen un amplio margen de autonomía para poder singularizar el modelo educativo en cada centro, en función de su contexto territorial y humano.

En definitiva, el centro docente posee autonomía en todo lo que no esté regulado como de obligado cumplimiento en el ámbito educativo. Las normas de desarrollo de las leyes, especialmente las de contenido curricular, establecen las competencias que debe alcanzar el alumnado al finalizar cada una de las etapas de la educación obligatoria, así como los objetivos de las mismas y los contenidos considerados básicos para lograr los dos elementos anteriores. Suelen incluirse también criterios de evaluación para los objetivos previstos en las distintas áreas que componen el campo de los contenidos (conceptos, procedimientos, actitudes). No obstante, en la legislación vigente en el momento en que se escribe este texto, no se marcan objetivos para las áreas curriculares, sino que se señalan criterios de evaluación (¿de qué, si no hay objetivos para evaluar?) y estándares de aprendizaje, muy concretos (demasiado) y numerosos.

\section{Diferentes enfoques de la autonomía}

En función de la actividad que puedan llevar a cabo diferentes organizaciones o empresas, la autonomía se ejerce en muy diversos campos, de acuerdo con las exigencias que plantea su progreso y mejora continuados. En el caso de las instituciones educativas, una autonomía total ${ }^{1}$, sin pertenencia a sistema alguno, llevaría aparejada la asunción absoluta en organización, diseño curricular, gestión económica, recursos humanos, equipamientos, instalaciones..., en definitiva, todo cuanto habitualmente está regulado y establecido por los sistemas educativos nacionales (o de cualquier carácter oficial-administrativo), dentro de los cuales se desarrollan las actuaciones docentes. No es el caso que nos ocupa. Como antes quedó apuntado, los centros privados poseen mayor autonomía que los encuadrados dentro de los conciertos educativos o los de carácter público; esta autonomía se refiere tanto a su modelo organizativo, su gestión económica o la selección de profesorado y de alumnado, si bien deben atenerse a las normas básicas que garanticen la calidad educativa establecida para todo el Estado, como son las titulaciones del profesorado, el respeto a las enseñanzas básicas y las instalaciones y equipamientos prescritos de forma general.

Los centros concertados mantienen su autonomía plena en la selección de profesorado, pero en todo lo demás deben atenerse a lo establecido para todos los centros sostenidos con fondos públicos (escolarización, gestión económica - con determinadas condiciones-, órganos de gobierno, etc.).

Para concretar los diferentes enfoques que puede adoptar la autonomía institucional en materia de educación, la encuadraremos en tres grandes ámbitos: autonomía pedagógica, autonomía organizativa y autonomía de gestión.

\subsection{Autonomía pedagógica}

Es la que resulta más interesante desde un punto de vista docente, pues permite tomar opciones diversas en relación con la definición y concreción del diseño curricular que, al fin, es lo que llega al aula y al estudiante.

Tomando como referencia los elementos del diseño curricular (Beltrán y San Martín, 2000; Zabalza, 2000; Casanova, 2015), hay que considerar que tanto las competencias clave, como los objetivos que se pretenden, suelen estar regulados de modo obligatorio por las Administraciones educativas, por lo que se prestan poco a las modificaciones por parte de los centros. Es posible ampliarlos, pero no reducirlos.

También aparecen contenidos básicos que deben respetarse, aunque sea posible ampliarlos o matizarlos de acuerdo con el contexto territorial en el que se produce la enseñanza: es una opción que los centros suelen tomar, elaborando temas propios de la zona y, por lo tanto, de especial interés para el alumnado. No obstante, los contenidos conceptuales son un "pretexto" tanto para trabajar el resto de contenidos (actitudes, valores..., y procedimientos) y para alcanzar los objetivos y competencias, referentes constantes para la evaluación y mejora de procesos. Son importantes, sin duda, por la carga cultural y cien-

1 Esta situación se daría en propuestas, experimentales en su momento, como fue la escuela de Summerhill, dirigida durante cincuenta años por A. S. Neill desde 1921 (Neill, 2014). 
tífica que proporcionan, pero sería necesario descargar de muchos de ellos los programas actuales de la educación obligatoria, pues la información se encuentra al alcance de una tecla de computadora y, sin embargo, las actitudes y procedimientos requieren de "presencia" y de relaciones personales, por lo que precisan de "tiempo" y orientación del docente para su consecución. Es una de las consecuencias que deberían adoptarse, como respuesta a la generalización de las TIC en la enseñanza. De hecho, durante el último semestre del curso 2019-2020, el profesorado se ha visto obligado a sintetizar los contenidos previstos curricularmente, dada la situación de pandemia, de confinamiento y, por lo mismo, de educación a distancia. Se ha realizado una selección imprescindible para poder seguir adelante y finalizar el curso de manera que el alumnado dispusiera de los aprendizajes realmente imprescindibles para poder continuar su formación sin problema en el curso siguiente. Quizá sea esta una buena base para comenzar a resituar tanto la cantidad de contenidos como las áreas o materias de aprendizaje. Muchas de las consideradas como fundamentales no lo han resultado tanto como otras, devaluadas en el sistema, pero que han sido imprescindibles para "sobrevivir" en tiempos de pandemia: plástica, música, educación física, teatro, cine, televisión, danza..., por no citar otras múltiples actividades que ni se contemplan curricularmente. Parece imprescindible repensar el currículo en lo referente a contenidos, pues se ha puesto de manifiesto la inoportunidad de muchos de ellos para la enseñanza en determinadas edades. Quizá habrá que ampliar la autonomía institucional, dando cabida a opciones también relacionadas con este elemento, que quizá ocupa un lugar más importante de lo que debiera en la realidad del aula.

En cuanto a las opciones metodológicas hay que destacar que es un elemento curricular en el que el centro tiene (desde siempre) una plena autonomía para decidir; supone elegir los métodos, estrategias, actividades o recursos didácticos idóneos para trabajar en el aula, de manera que se ofrezcan caminos diversificados al alumnado, respondiendo así a sus estilos y ritmos de aprendizaje, motivaciones, intereses, capacidades o talentos, contexto familiar y social..., y puedan llegar a las metas previstas. Solamente estará condicionado por las competencias y objetivos que se quieran lograr, pues en función de ellos habrá que implementar unas u otras estrategias: para conseguir ciudadanos obedientes y callados, lo mejor será que estudien la lección, escuchen al maestro y repitan lo que ambos dicen; para formar ciudadanos demócratas y participativos, habrá que utilizar el trabajo por proyectos, basado en problemas, en retos, la gamificación, el aula invertida..., en fin, caminos adecuados para aprender a vivir, durante la ruta, viviendo como más tarde se hará en la sociedad extraescolar.

En este campo, el centro deberá decidir dos tipos de opciones para implementar el proyecto curricular del centro:

- Opciones generales para un determinado ciclo, curso o etapa educativos.

- Opciones específicas para cada área o materia curricular.

En el primer caso, se trata de acordar modelos metodológicos aplicables a cualquier área o materia, como pueden ser el trabajo por proyectos, aprendizaje basado en retos o problemas, unidades didácticas, exposiciones magistrales, debates, coloquios, mapas mentales o conceptuales, gamificación, trabajo cooperativo, talleres, asambleas..., y otros numerosos de los que disponemos en la actualidad. Estos serán utilizados en el aula por todo el profesorado que intervenga en un mismo grupo de alumnos, en las situaciones que consideren pertinentes, pero constituyendo el núcleo metodológico diversificado por el que se opta, no manteniendo un único modelo, quizá superado y obsoleto para el alumnado de nuestro tiempo.

En el segundo caso, se tratará de que el profesorado de cada área o materia, selecciones los métodos, actividades o recursos didácticos más idóneos para su enseñanza y aprendizaje. Es evidente que matemáticas, ciencias sociales o lengua y literatura precisan de estrategias, actividades y recursos diferentes para su aprendizaje.

Es este un ámbito fundamental para lograr una educación de calidad, pues en él se deciden las actuaciones más apropiadas para cada alumno o alumna y, por ello, será desde el que se marque la vía que le conduzca a su mejor formación. Obviamente, la autonomía institucional es total para tomar las decisiones metodológicas, pues las Administraciones cuando regulan el currículo no "obligan" a aplicar ninguna metodología en particular, sino que, como máximo, publican orientaciones metodológicas que, como bien indica su nombre, solo son indicaciones o apoyos para que los docentes decidan los que mejor se adapten a su proyecto. 
Por lo que se refiere a la evaluación de aprendizajes, habitualmente se marcan como obligatorios unos criterios para las distintas áreas/materias curriculares. Además, se establecen los tiempos y formas de comunicación de resultados a las familias, al igual que el formato del expediente o historial académico del alumnado. En los últimos años, han aparecido las pruebas externas de evaluación, que se llevan a cabo por parte de las Administraciones en determinados momentos de las etapas educativas y las pruebas de carácter internacional, que también se han generalizado en el sistema. En estos puntos no es posible ejercer la autonomía institucional, salvo en los modelos de informes a las familias, que se ajustarán a lo que se haya decidido en el proyecto educativo de cada centro.

Donde es posible e importante ejercer la autonomía es en los modelos y procedimientos de evaluación de aprendizajes en las aulas; es decir, en optar por modelos superados, como puede ser el examen como único instrumento evaluador, o practicar técnicas y registros variados que permitan valorar el trabajo real del alumnado, dentro y fuera del aula (Casanova, 2019). Hay que concluir que, si la opción educativa se ha decantado por la inclusión, todos los elementos curriculares tendrán que colaborar en conseguirla, no en minarla mediante prácticas incorrectas, que impidan su buena marcha. La autonomía en cuanto a la evaluación de aprendizajes que realiza el profesorado es casi absoluta, ya que el modo de valorar las actividades escolares depende, exclusivamente, del docente. Hay que abogar, no obstante, porque el modelo de evaluación sea común para todo el centro, pero, en caso de no conseguir este consenso, bueno será aplicar un modelo cooperativo de evaluación, frente al tradicional que estimula la competitividad y las comparaciones inaceptables entre unos y otros alumnos.

\subsection{Autonomía organizativa}

Por lo que se refiere a la organización institucional, conviene recordar que, si se pretende aplicar un currículo flexible, también habrá que dotar de esta característica al modelo organizativo que se practique. Un currículo cerrado se "imparte" sin problema en grupos inamovibles, con horarios fijos, trabajos individuales del alumnado, independencia de cada profesor para trabajar su materia sin coordinarse con el resto del claustro, etc. Sin embargo, si lo que se pretende es adecuar el sistema al estudiante, la accesibilidad mediante la flexibilidad organizativa resulta imprescindible para lograr el objetivo.

Es preciso abogar, en este punto, para que se ejerza la autonomía mediante la asunción de distintos modelos de agrupamientos, modelos cooperativos y colaborativos del trabajo entre el profesorado, horarios coordinados para poder intercambiar profesores y alumnos, creación de centros de recursos institucionales disponibles para el conjunto de docentes, el ejercicio de una dirección participativa delegando las funciones pertinentes, el estímulo de la fluidez de la información entre todos los miembros de la comunidad escolar, la promoción de la intervención positiva de las familias en actuaciones del centro, el concierto de actividades diversas con entidades del entorno..., y un largo etcétera que permitirán al centro contar con recursos de todo tipo, humanos y materiales, para favorecer el enriquecimiento de los procesos educativos que en él surgen diariamente (Cano, 2003).

No se puede olvidar tampoco la importancia que la arquitectura, la distribución y disposición de los espacios escolares, tiene para facilitar o no este modelo organizativo flexible. La unión de varios grupos para realizar una actividad concreta exigirá disponer del espacio que lo permita. Más aún si el modelo organizativo parte ya del trabajo permanente, individual y en grupo, de grandes agrupamientos a lo largo del curso, trabajando con más de un profesor simultáneamente. En estos momentos se cuenta con múltiples opciones arquitectónicas y de equipamiento, que permiten variar los espacios sin mayor dificultad.

La autonomía institucional en este campo es amplia, pues no hay indicaciones obligatorias en la normativa vigente, aunque en muchas ocasiones (demasiadas), por los modelos generalizados que se observan en nuestro sistema, bien se podría afirmar que existe una norma cerrada e infranqueable. En definitiva, se concluye en la necesidad de ejercer la autonomía en el campo de la organización, con objeto de que esta constituya un factor decisivo - como lo es- para la implementación adecuada del diseño curricular inclusivo que haya concretado el centro a través de sus órganos de participación y gobierno.

\subsection{Autonomía de gestión}

Por último, cabe citar la autonomía de gestión económica, que colaborará eficazmente en la utilización de los recursos materiales que debe disponer el centro para su buen funcionamiento. 
Es otra modalidad de ejercicio autonómico que permite decidir la inversión que, en cada circunstancia, precise el centro para contar con los materiales que necesite. Una comisión con representantes de los diferentes sectores de la comunidad educativa se encargará de esta gestión autónoma, rindiendo cuentas en los momentos prescritos.

Aunque pueda parecer menos importante o, quizá, menos compleja la tarea de gestionar la economía institucional, es conveniente destacar su relevancia, dado que en múltiples ocasiones la no disponibilidad de este recurso flexible puede impedir la realización de actuaciones pedagógicas fundamentales para el proyecto previsto.

En definitiva, la conjunción de los tres enfoques de autonomía que se presentan constituye un todo inseparable que permitirá llevar adelante la importante planificación que cada comunidad decida.

\section{Exigencias para el ejercicio responsable de la autonomía}

En general, existe aquiescencia en la necesidad y conveniencia de contar con autonomía institucional en los centros docentes, para que estos puedan ejercer las funciones que se les exigen $y$, también, responder ante la sociedad de la calidad educativa conseguida con su trabajo profesional. Ante esta afirmación, hay que dejar constancia de que en demasiados centros prefieren "obedecer" lo que establezca la Administración, antes que tomar las propias decisiones bajo su exclusiva responsabilidad. Quizá por comodidad, quizá por inseguridad o por falta del clima adecuado de convivencia..., no se atreven a decidir. Esto supone un grave problema, dado que la autonomía está transferida y, si los centros no la asumen, nadie va a decidir por ellos.

En otro orden de cosas, para que el ejercicio de la autonomía sea responsable, se plantean tres requisitos indispensables: formación actualizada de los equipos directivos y del profesorado, liderazgo e implementación de evaluación interna institucional

\subsection{Formación permanente y actualizada}

En la actualidad, no solo se pide al docente que domine científica y didácticamente la materia o áreas que debe trabajar, sino que se le exigen otros conocimientos y competencias relacionados con las tareas que se acaban de exponer en este texto: organización del aula, programación, dominio tecnológico, técnicas e instrumentos de evaluación, coordinación colegiada, relación con las familias y el entorno, atención a la diversidad del alumnado... Por ello, no basta con la formación inicial que recibe para disponer de la titulación requerida, sino que, contando con los avances continuos que aparecen en todos los campos técnico-pedagógicos, precisa de una actualización permanente también en los ámbitos complementarios de su trabajo didáctico. Es compleja la educación en la sociedad que vivimos y hay que mantenerse en permanente actitud de aprendizaje para ofrecer las respuestas que se esperan de la educación institucional (Elliott, 1990).

Como es evidente, si este tipo de formación es necesaria para todo el profesorado, resulta absolutamente imprescindible para la dirección, la jefatura de estudios o la secretaría del centro. Equipo directivo que debe poseer un dominio específico tanto de administración, como de gestión (también del conocimiento, por supuesto), de diseño, de evaluación... Además de contar con un buen nivel de competencias relacionales y de equilibrio emocional, pues de lo contrario servirá de poco el conocimiento racional si no va acompañado de una personalidad estable y con facilidad para la comunicación.

En el momento actual es imposible obviar la importancia adquirida en pocos meses del dominio informático por parte del profesorado y de los directivos en educación. La situación de confinamiento ya aludida ha convertido las TIC en el elemento clave para continuar la formación, en este caso a distancia, obligada por el cierre de los centros. Se han convertido en el eje que ha permitido finalizar el curso 2019/2020 de un modo razonable. En los comienzos del 2020/2021 continúa siendo protagonista, dada la situación sanitaria existente: quizá sea un modelo híbrido de enseñanza el que vaya a predominar, lo cual exige mantener el perfeccionamiento docente en el campo tecnológico y ejercer, así, los procesos educativos con nuevos medios y metodologías. 


\subsection{Liderazgo inclusivo}

El trabajo individual del profesorado en el aula es importante, no cabe la menor duda. Pero ese trabajo no rinde como debiera si no forma parte de un proyecto común y de unas opciones compartidas con el conjunto del equipo profesional. Y la creación y pervivencia de ese proyecto depende del liderazgo de su dirección o equipo directivo (Bolívar, 2019).

La importancia de disponer de un equipo líder en la institución educativa es la clave para su buen funcionamiento y la obtención de los resultados previstos. Esta afirmación es más contundente cuando nos referimos a los centros públicos, que no poseen autonomía para la selección de personal y, por lo tanto, requieren un mayor liderazgo directivo para aunar los perfiles del diferente profesorado que se incorpora a ellos (Murillo y Hernández, 2014).

Con el paso del tiempo y los cambios sociales ya señalados, es obligado reconocer que también la noción de líder se ha modificado, teniendo especial interés el liderazgo inclusivo (Ryan, 2017), que trata de promover e impulsar los proyectos acordados a través de la participación de todos los implicados en la acción, aprovechando los talentos y capacidades de todos, creando sinergias de funcionamiento proactivo y no liderando en solitario, con la fuerza del carácter personal y la autoridad o, en su caso, el autoritarismo. Puede resultar más complejo, pero siempre resulta más satisfactorio y conduce a mejores resultados, pues compromete a la comunidad completa como protagonista de los procesos $\mathrm{y}$, por lo tanto, también de las metas alcanzadas.

En función de este planteamiento, resulta fundamental que el líder sea capaz de:

- Construir relaciones ágiles, flexibles y auténticas

- Potenciar la comunicación empática

- Crear entornos seguros

- Estimular la creatividad y la innovación

- Promover la autonomía de los miembros de la comunidad

- Favorecer el ejercicio responsable de las acciones individuales y grupales

- Promocionar las competencias interculturales en el centro

- Valorar a cada persona descubriendo sus valores y talentos

- Desagregar el conjunto de tareas realizables en el centro, para asignarlas a las personas idóneas para su puesta en práctica

Evidentemente, un líder inclusivo será fácilmente reconocido y aceptado por todos y, en consecuencia, constituirá la mejor garantía de comprometer a toda la comunidad escolar en la planificación común y de responsabilizarla en su implementación inclusiva.

\subsection{Evaluación interna}

A más autonomía pedagógica, mayor necesidad de evaluación interna. Si la posibilidad de tomar opciones autónomamente es esencial para conseguir la calidad educativa requerida, esta no podrá lograrse sin mantener un proceso continuo de evaluación interna del centro; es decir, sin tener diseñado un modelo evaluativo que permita controlar y ajustar paso a paso los aciertos y las disfunciones que se presenten durante el transcurso de la acción docente y de gestión en general.

Un modelo de evaluación institucional formativa (Casanova, 2007), cuya finalidad principal será valorar cómo se desenvuelven los procesos de funcionamiento previstos, de manera que, mediante las reuniones oportunas con los agentes evaluadores de cada factor evaluado, se vaya decidiendo cómo continuar para llegar al final del curso con los objetivos cubiertos. Si se trabaja con personas, hay que ser consciente de que el tiempo no se recupera. El trabajo de gestión y de docencia en los centros se dirige a la mejor educación de las generaciones escolarizadas y estas no pueden permitirse perder un año tras otro esperando la 
toma de medidas pertinente para mejorar la calidad educativa en el centro. Es responsabilidad del equipo directivo, del claustro y, al fin, de la comunidad completa, que las disfunciones que aparezcan se resuelvan de inmediato, al igual que se promuevan y generalicen los aspectos de funcionamiento que se identifiquen como puntos fuertes de la institución. Evaluar, así, para destacar lo positivo y corregir lo negativo.

Como muestra de un posible registro de seguimiento para realizar esta evaluación, puede consultarse la figura 1, en la que se marcan los indicadores que se pretenden en la primera columna; en la segunda se refleja cómo está la realidad del centro en relación con el mismo (más o menos conseguido); en la tercera parece la distancia que se debe recorrer para llegar al cumplimiento del indicador señalado y en la cuarta se reflejarán las acciones precisas para conseguir el funcionamiento institucional contemplado en el proyecto educativo y curricular.

Figura 1

Modelo para la evaluación interna del centro

Indicador Realidad del funcionamiento del centro $\begin{gathered}\text { Distancia entre el } \\ \text { indicador } y \text { la realidad } \mathrm{I} / \mathrm{R}\end{gathered} \quad$ Proyecto de mejora

Nota. Elaboración propia.

El contar con datos de evaluación interna permitirá, además, contrastarlos con las evaluaciones externas que ahora se practican, tanto a nivel comunitario, como nacional e internacional. Será la mejor forma de conocer con mayor objetividad el funcionamiento real del centro y de tomar las medidas oportunas para su mejora.

\section{A modo de conclusión}

Como puede comprobarse a través del texto precedente, por la experiencia de múltiples instituciones y la obtenida mediante la bibliografía consultada, el dotar de autonomía de funcionamiento a los centros docentes resulta una propuesta no solo razonable, sino exigible a las Administraciones, pues sienta la base de una práctica responsable y comprometida del personal que compone y define las comunidades educativas, que asumen un papel eminentemente protagonista del nivel de convivencia, los procesos de gestión y aprendizaje $y$, por consiguiente, de los resultados que se obtienen cada año académico en la institución, ofreciendo también la posibilidad de mejora permanente de cada uno de estos elementos que, sin duda, progresarán de modo continuo mediante las opciones diversas que se adopten en cada caso.

Autonomía y evaluación interna constituyen dos componentes inseparables, que deben reforzarse entre sí y convertirse en la clave de la calidad educativa exigible en cada momento de la historia, modificando de forma continua las actuaciones docentes y directivas.

Es muy escasa la cultura de evaluación existente en la realidad educativa española, especialmente en lo que se refiere a la revisión y reflexión institucional generalizada y sistemática en los centros docentes, quizá debido, sobre todo, a la mala interpretación y utilización que se hace de la misma en otros ámbitos de aplicación. Será muy importante, por ello, reforzar la formación de directivos y profesorado en este campo, si se quiere avanzar en un modelo de autonomía pedagógica institucional que ofrezca resultados positivos, responsables y con garantías de avance continuo en la mejora de la calidad que se ofrece al alumnado y a la sociedad.

Por otra parte, también hay que reseñar que, a pesar de los años transcurridos desde la dotación de autonomía a las instituciones educativas por parte de la Administración, esta no se ejerce plenamente, posiblemente por la falta de formación y seguridad en las decisiones que puedan adoptarse desde los equipos directivos correspondientes. Se trata de una situación similar a la descrita en el párrafo anterior y con la misma conclusión: resulta imprescindible reforzar la formación de directivos en las disciplinas directamente relacionadas con el ejercicio de su desempeño profesional. 


\section{Referencias}

Ainscow, M. (2014). Desarrollo de escuelas inclusivas. Ideas, propuestas y experiencias para mejorar las instituciones escolares. Narcea.

Beltrán, F. y San Martín, A. (2000). Diseñar la coherencia escolar. Morata.

Bolívar, A. (2019). Una dirección escolar con capacidad de liderazgo pedagógico. La Muralla.

Cano García, E. (2003). Organización, calidady diversidad. La Muralla.

Casanova, M. A. (2007). Evaluación y calidad de centros educativos. La Muralla.

Casanova, M. A. (2015). Diseño curricular e innovación educativa. La Muralla.

Casanova, M. A. (2017). Educación inclusiva en las aulas. La Muralla.

Casanova, M. A. (2019). Manual de evaluación educativa. La Muralla.

Davis, G.A. y Thomas, M.A. (2007). Escuelas eficaces y profesores eficientes. La Muralla.

Elliott, J. (1990). La investigación-acción en educación. Morata.

Morin, E. (2001). Los siete saberes necesarios para la educación del futuro. Paidós Studio.

Moya, J. (2015). La educación como derecho. Bases para un consenso razonable. La Muralla.

Murillo, F. J. y Hernández, R. (2014). Liderazgo escolar, un elemento clave en la promoción de la educación para la justicia social. Revista Internacional de Educación para la Justicia Social, 3(2), 5-10.

Neill, A. S. (2014). Summerhill. Fondo de Cultura Económica.

Redondo, N. (2011, 18 de marzo). ¿El pasado siempre vuelve? El Economista.

Ryan, J. (2017). Un liderazgo inclusivo para las escuelas. En J. Weinstein (Ed.), Liderazgo inclusivo en la escuela: Nueve miradas (pp. 177-204). Ediciones Universidad Diego Portales.

VV.AA. (2009). La autonomía de los centros educativos. Consejería de Educación de la Comunidad de Madrid.

VV.AA. (2011). Actuaciones de éxito en las escuelas europeas. Ministerio de Educación de España.

Zabalza, M. A. (2000). Diseño y desarrollo curricular. Narcea.

\section{Breve CV de la autora}

\section{Ma Antonia Casanova}

Profesora de la Universidad Camilo José Cela y de la Universidad Villanueva, de Madrid. Dirige el Instituto Superior de Promoción Educativa. A lo largo de su carrera se ha desempeñado como supervisora de educación, ocupando diferentes puestos en la Administración educativa: Subdirectora general de Educación Especial y Atención a la Diversidad (Ministerio de Educación) o Directora general de Promoción Educativa (Comunidad de Madrid). Colabora en distintas publicaciones periódicas y dirige la Colección de Pedagogía "Aula Abierta. Entre sus publicaciones, cabe destacar: Manual de evaluación educativa, Educación inclusiva en las aulas, Educación de futuro: textos para el debate. Email: acasanova@ucjc.edu

ORCID ID: https://orcid.org/0000-0003-0511-7809. 